\title{
Large Aperture Kinoform Phase Plates in Fused Silica for Spatial Beam Smoothing on Nova and the Beamlet Lasers
}

\author{
M. C. Rushford, S. N. Dixit, I. M. Thomas,
}

A. M. Martin, and M. D. Perry

This paper was prepared for submittal to 2nd Annual International Conference on Solid-State Lasers for Applications to Inertial Confinement Fusion Paris, France

October 22-25, 1996

March 14, 1997

This is a preprint of a paper intended for publication in a journal or proceedings. Since changes may be made before publication, this preprint is made available with the understanding that it will not be cited or reproduced without the permission of the author. 


\title{
Large aperture kinoform phase plates in fused silica for spatial beam smoothing on Nova and the Beamlet lasers
}

\author{
M. C. Rushford, S. N. Dixit, I. M. Thomas, A. M. Martin and M. D. Perry \\ Lawrence Livermore National Laboratory \\ Livermore, California 94550
}

Tel: (510) 4237321 FAX (510) 422-5537 E-mail: dixit1@1lnl.gov

\section{INTRODUCTION}

It is now widely recognized that spatial beam smoothing (homogenization) is essential in coupling the laser energy to the inertial confinement fusion (ICF) targets ${ }^{1}$. For the indirect drive approach ${ }^{1}$ to ICF, it is desirable to distribute the laser energy into a uniformly speckled profile that has a flat-top super-Gaussian envelope (8th power or higher) and contains greater than $95 \%$ of the energy inside the super-Gaussian profile. Spatial smoothing is easily achieved by introducing a binary random phase plate (RPP) in the beam. This produces a homogenized far-field pattern which consists of an overall envelope function determined by the RPP element superimposed with a fine scale speckle pattern arising due to the interference among the various RPP elements. Although easy to fabricate and currently in routine use in many fusion laboratories ${ }^{2}$, the binary RPPs do not meet the ICF requirements stated above since the far-field intensity profile is restricted to essentially an Airy function containing only $84 \%$ (an upper limit) of the energy inside the central spot. Approaches using lenslet arrays (refractive or diffractive) ${ }^{3,4}$ have limited use since they operate in the quasi-far-field and have a short depth of focus.

The limitations of the RPPs can be overcome by relaxing the binary phase constraint. We have recently presented ${ }^{5}$ continuously varying phase screens for tailoring the focal plane irradiance profiles. Called kinoform phase plates (KPPs), these phase screens offer complete flexibility in tailoring the focal plane envelope and, at the same time, increasing the energy efficiency inside the focal spot.

In this paper we discuss the design and fabrication of such kinoform phase plates in fused silica for spatial beam smoothing on the Nova and the Beamlet lasers. Since the phase plates are used at the end of the laser chain, KPPs on Nova and Beamlet have to be fabricated on large aperture optics $(65-\mathrm{cm}$ diameter and $40-\mathrm{cm}$ square substrates respectively). The following section presents a brief review of process for designing the KPPs. In section 3, we discuss the fabrication of large aperture KPPs in fused silica substrates. The optical performance of these KPPs is discussed in section 4 which is followed by a summary.

\section{DESIGNING FULLY CONTINUOUS KPPs}

As mentioned in above, generation of superGaussian far-field profiles which contain a high percentage of the incident energy requires the relaxation of the binary phase 
assumption used in designing the RPPs. Thus the KPP design problem amounts to constructing a continuous phase screen which produces the desired far-field irradiance profile. In a focusing geometry, the electric fields at the lens and at the focal plane are related to each other by a Fourier transform. Since one is not interested in the phase of the electric field in the focal plane, it can be treated as a free parameter. This phase freedom in the far-field allows one to use algorithms based on phase retrieval to construct the required KPP phase screens. A very powerful algorithm in this regard is the Gerschberg-Saxton (GS) algorithm $^{5}$. We have applied ${ }^{6}$ the Gerschberg-Saxton algorithm to design KPPs that produce arbitrary and quite complicated focal plane irradiance profiles. The flow of the algorithm is illustrated in Fig. 1. Here, one begins with the known near-field intensity profile and a random phase screen. The phase screen is iteratively improved upon by the repeated propagation between the near-field and the far-field planes and the application of the near-field and the far-field constraints at each iteration step. These constraints typically consist of replacing the intensity profile with the desired profile while leaving the phase unchanged. The algorithm is rapidly convergent and phase screens can be generated for producing arbitrary focal plane intensity profiles.

Inspite of the robustness of the GS algorithm, it has a serious limitation when used in a conventional manner. If the algorithm is launched with an initial random phase screen, it is observed that the converged KPP phase has many open ended $2 \pi$ jumps in addition to the closed loop $2 \pi$ jumps. The algorithm in reference 6 stagnates at the open ended $2 \pi$ phase discontinuities and further iterations are unable to remove them.

The difficulty with $2 \pi$ lines is twofold. A phase screen containing such $2 \pi$ lines cannot be unwrapped into a continuous phase screen. The $2 \pi$ lines represent branch cuts with phase singularities at the ends. Further, the rapid variation of the phase around the ends of the $2 \pi$ lines leads to large modulations in the near-field beam following the phase plate and to far-field energy losses due to large-angle scattering ${ }^{7}$. A fully continuous phase screen, on the other hand, minimizes near-field intensity modulation following the phase plate and increases the energy concentration inside the central spot due to the elimination of large angle scattering. Because of these advantages, it is desirable to generate fully continuous phase screens for ICF beam smoothing applications.

We have recently presented ${ }^{8}$ an improvement to the GS algorithm which enables us to generate fully continuous phase screens for generating high order superGaussian focal plane irradiance profiles. The principal difference of the new method compared to the conventional GS approach is in starting the iteration cycle with a continuous phase profile and preserving its continuous nature throughout the iteration process. This is accomplished by a very slow, adiabatic modification of the far-field profile with each iteration step. Figure 2a shows an example of such a fully continuous phase screen constructed using the new algorithm. The far-field irradiance profile produced by it is shown in Fig. $2 \mathrm{~b}$ and it closely resembles a 12th power superGaussian.

Using this algorithm, we have designed fully continuous phase screens for use on the Beamlet laser and on the Nova laser. The converged phase screens are displayed in Figures $3 \mathrm{a}$ and $3 \mathrm{~b}$ respectively with the corresponding far-field intensity distributions for 
unaberrated beams shown in figures $4 \mathrm{a}$ and $4 \mathrm{~b}$. Both of these phase plate designs are for use at $351 \mathrm{~nm}$. For aberrated beams, the overall far-field is given by a convolution of the KPP far-field for an unaberrated beam and the far-field of the aberrated beam. If the KPP spot is considerably larger (by a factor of 2 or higher) than the beam spot, then the effect of the beam aberrations is to slightly blur the spot and degrade the flatness of the envelope. On the other hand, if the two spot sizes are comparable, the KPP spot shape and size can be severely degraded. Since the beam aberrations for high power laser are not known a priori and often vary from shot to shot, some allowance has to be made in the KPP design to allow for the broadening of the focal spot produced by the KPP due to the beam aberrations. The Beamlet KPP is designed to produce a $350 \mu \mathrm{m}$ diameter circular superGaussian spot when used with a $7 \mathrm{~m}$ focal length lens. The Nova KPP is designed to produce an elliptical spot with $\sim 400 \mu \mathrm{m}$ diameter major axis and $\sim 260 \mu \mathrm{m}$ diameter minor axis when used with a $3 \mathrm{~m}$ focal length lens. The ellipticity parameters were chosen so that the KPP spot would appear nearly circular when viewed in the plane of the laser entrance hole for the hohlraum (at 50 degrees to the normal) on Nova. The far-field profiles in each case contains greater than $98 \%$ of the incident energy.

\section{FABRICATION OF THE KPPs IN FUSED SILICA}

In this section, we shall discuss fabrication of the Beamlet and the Nova phase plate designs discussed in the previous section on fused silica substrates. Laser damage considerations imply that the phase plates have be fabricated in fused silica substrates and located following the final focus lens in the beam path. This requires that the phase plates have to fabricated at large aperture sizes. The aperture size for the Beamlet KPP is $40 \times 40 \mathrm{~cm}$ while that for the Nova KPP is 65-cm diameter circle. To achieve high focusing efficiency, it is desirable to fabricate the KPPs as a continuous profile. However, methods involving gray scale patterning of a photoresist layer and transfer etching this gray scale pattern into fused silica by dry etching are not scalable to our KPP fabrication because of the large aperture sizes involved. It is our opinion that the process involving the patterning of an epoxy layer ${ }^{9}$ is not adequate for our applications because of the insufficient damage threshold of the epoxy layers.

Lithographic approach combined with wet etching is an alternative approach to fabricating the kinoform phase plates on large apertures. Here the fully continuous phase screen (several waves deep) is first re-wrapped into a one-wave deep, mod $2 \pi$ phase profile and this wrapped kinoform phase profile is quantized into sixteen discrete levels. Quantization of the phase profile to sizteen levels leads to a $1 \%$ reduction in the focussing efficiency of the phase plate. The discretized 16 level phase structure is fabricated in fused silica by a successive superposition of four binary masks and a differential etching of the KPP substrate at each step. In recent years, we have fabricated several binary RPPs on large aperture (upto $80 \mathrm{~cm}$ diameter) fused silica substrates by this process. This experience has lead us to pursue the lithographic approach for KPP fabrication for high power ICF applications.

In the lithographic approach to KPP fabrication, maintaining a high diffraction efficiency in the focal spot requires the production of precise masks and an accurate overlay 
of these masks on the KPP substrate. The binary photomasks required for the KPP fabrication were patterned in a chrome layer coated over a fused silica substrate using the large aperture photoplotter developed at our laboratory. We chose to write the masks on fused silica substrates to minimize errors due to thermal effects. The available plotting area for the photoplotter is about $1 \mathrm{~m} \times 1 \mathrm{~m}$ square. The pixel positioning and the pixel size are accurate to about $0.5 \mu \mathrm{m}$ each over the entire plotting area. Beamlet KPP masks had a pixel size of about $867 \mu \mathrm{m}$ where as the Nova KPP masks were written with a $700 \mu \mathrm{m}$ pixel. Pixelation of the phase profile gives rise to a far-field diffraction envelope characteristic of a single pixel. The secondary maxima take away some of the energy from the central spot and further reduce the KPP efficiency. The loss of energy into the other diffraction orders is related to the ratio of the KPP spot size to the separation of the secondary maxima. It can be minimized by writing the masks with smaller and smaller pixels. However, going to smaller and smaller pixels increases the relative effect of misalignment of various masks and edge degradation during the lithographic steps. The edge definition through the sequence of the lithographic steps mentioned above erodes to about $1.5 \mu \mathrm{m}$ per mask step. The etch depths in fused silica were controlled to $10-15 \mathrm{~nm}$ at each etch step. We estimate that the various quantization and fabrication errors amount to approximately $2 \%$ efficiency loss for the Beamlet KPP and $3-4 \%$ loss for the Nova KPP.

Using the process described above, we have fabricated a large aperture KPP for use on the Beamlet laser and ten KPPs for use on each of the ten Nova beamlines. The optical performance of these phase plates was evaluated by examining the far-field intensity distribution produced by the KPPs when illuminated with an unaberrated $30-\mathrm{cm}$ diameter beam. The observed far-fields are displayed in figure 5 and 6 for the Beamlet and the Nova KPPs. The observed far-field profiles closely resemble the predicted ones shown in figure 4. The efficiency of the KPPs was measured by measuring the energy passing through different sized pinholes located in the focal plane. The phase plates were anti-reflection coated with a sol-gel solution to minimize Fresnel reflection losses. The measured efficiencies are compared in figures 7 and 8 with the theoretical efficiencies adjusted for quantization, pixelation and mask misalignment losses. Measured efficiencies are in excellent agreement with the predicted theoretical efficiencies. Although the efficiencies and the far-field spot are shown for only one Nova KPP, the spot shapes and the diffraction efficiencies are very similar for all the ten KPPs fabricated for Nova.

Finally, the KPPs were fielded on the Beamlet and the Nova lasers. The observed focal plane irradiance profile are shown in figures 9 and 10. The focal spots produced by the KPPs on Nova and Beamlet are distorted because of the aberrations on these beams. It seems, however, that the overall shape of the spot still seems to be determined by the KPP with the beam aberrations altering the finer features of these spots. This implies that the beam aberrations play a somewhat subsidiary role in the focal plane irradiance profile. This increases our confidence in the usefulness of the KPPs for spatial beams smoothing on high power fusion laser systems. 


\section{SUMMARY}

In summary, we presented the design and fabrication of large aperture kinoform phase plates for producing homogenized focal plane intensity profiles. The KPP phase screens are fully continuous and are designed using a new iterative algorithm recently proposed by us. The large aperture KPPs were fabricated in fused silica using lithographic processes and wet etching. The observed far fields for unaberrated beams are similar to the predicted ones and the measured efficiencies are in close agreement with theoretical predictions. The KPPs are approximately 95\% efficient. Finally, these KPPs were fielded on the Nova and the Beamlet laser systems. The recorded focal spots, although somewhat blurred by the beam aberrations, still resemble to a large extent the spots produced by the KPPs.

\section{ACKNOWLEDGEMENT}

This work was performed under the auspices of the U. S. Department of Energy by the Lawrence Livermore National Laboratory under Contract No. W-7405-ENG-48.

\section{REFERENCES}

1. J. D. Lindl, R. L. McCrory and E. M. Campbell, "Progress toward ignition and burn propagation in inertial confinement fusion," Physics Today 4532 (1992)

2. Y. Kato, K. Mima, N. Miyanaga, S. Arinaga, Y. Kitagawa, M. Nakatsuka and C. Yamanaka, "Random phasing of high-power lasers for uniform target acceleration and plasma instability suppression," Phys. Rev. Lett. 531057 (1984).

3. X. Deng, X. Liang, Z. Chen, W. Yu and R. Ma, "Uniform illumination of large targets using a lens array," Appl. Opt. 25377 (1986).

4. R. M. Stevenson, M. J. Norman, T. H. Bett, D. A. Pepler, C. N. Danson and I. N. Ross, "Binary zone plate arrays for the generation of uniform focal profiles," Opt. Lett. 19363 (1994)

5. R. W. Gerchberg and W. O. Saxton, "A practical algorithm for determination of phase from image and diffraction plane pictures," Optik, 35, 237 (1972)

6. S. N. Dixit, J. K. Lawson, K. R. Manes, H. T. Powell and K. A. Nugent, "Kinoform phase plates for focal plane irradiance profile control," Opt. Lett. 19417 (1994).

7. Y. Lin, T. Kessler and G. Lawrence Opt. Lett paper

8. S. N. Dixit, M. D. Feit, M. D. Perry and H. T. Powell, "Designing fully continuous phase screens for tailoring focal-plane irradiance profiles," Opt. Lett. 211715 (1996). 


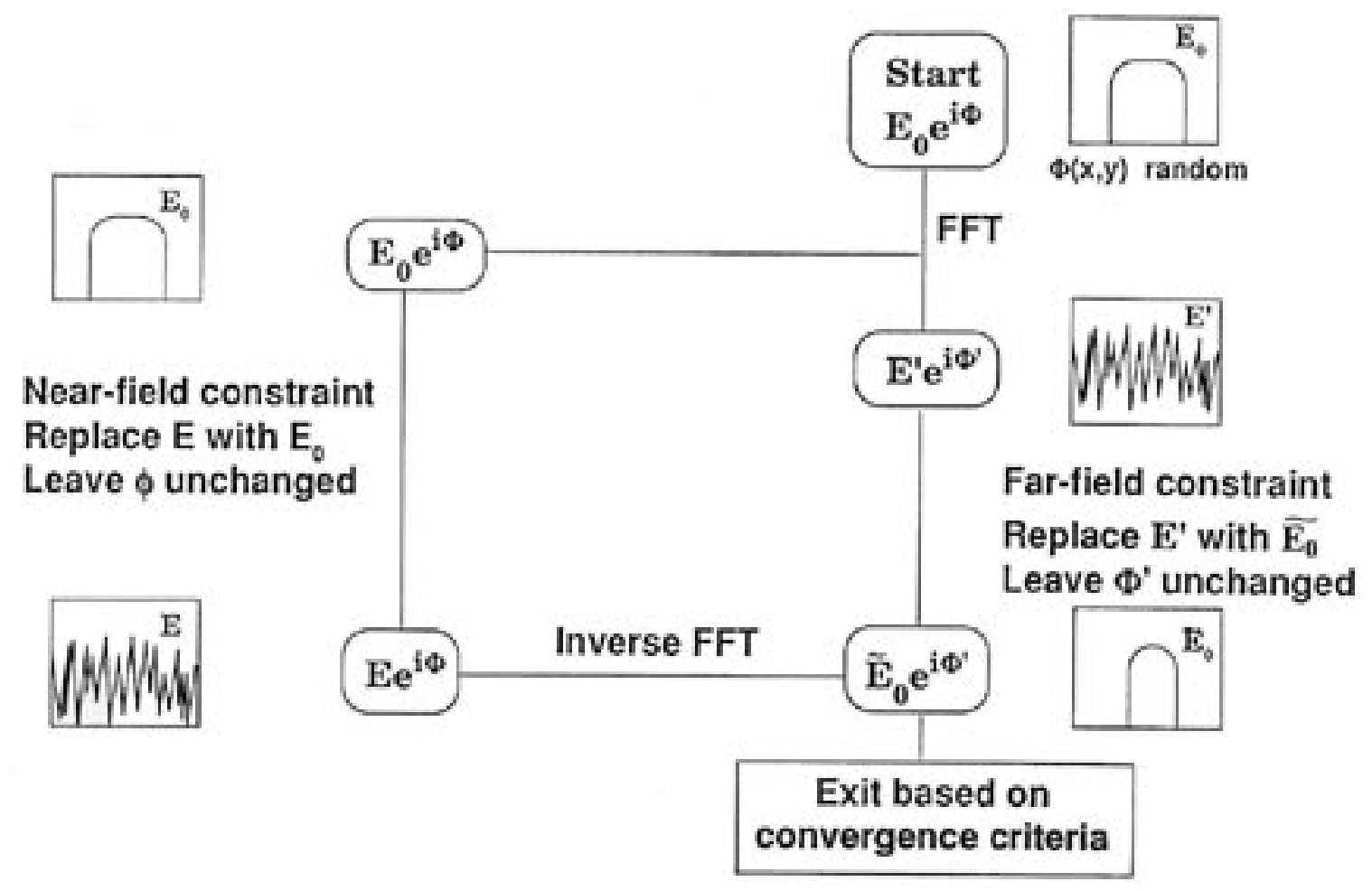

Figure 1. Schematic of the flow of the Gerschberg-Saxton iterative algorithm for designing kinoform phase plates

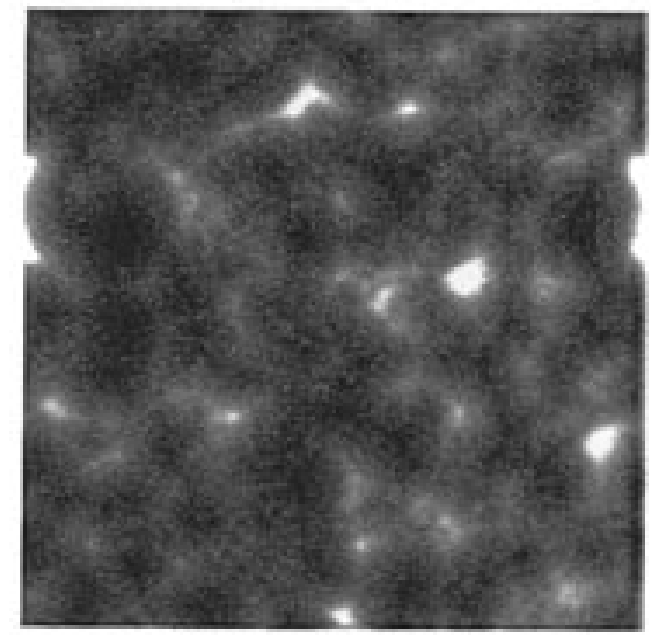

(a)

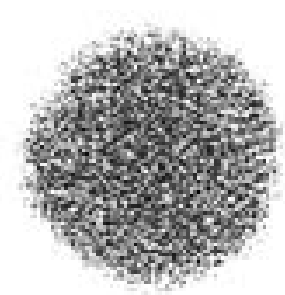

(b)

Fig 2. (a) An example of a continuous phase screen. The gray scale (linear from black to white) corresponds phase values from 0 (black) to 43 radians (white).

Fig. 2 (b) Far field pattern produced by the phase screen in figure 2a. The envelope can be approximated by a 12 th power superGaussian. 


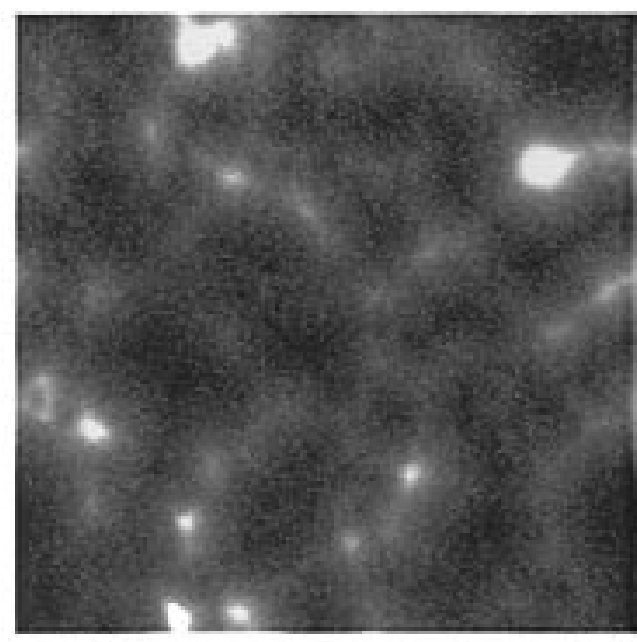

(a)

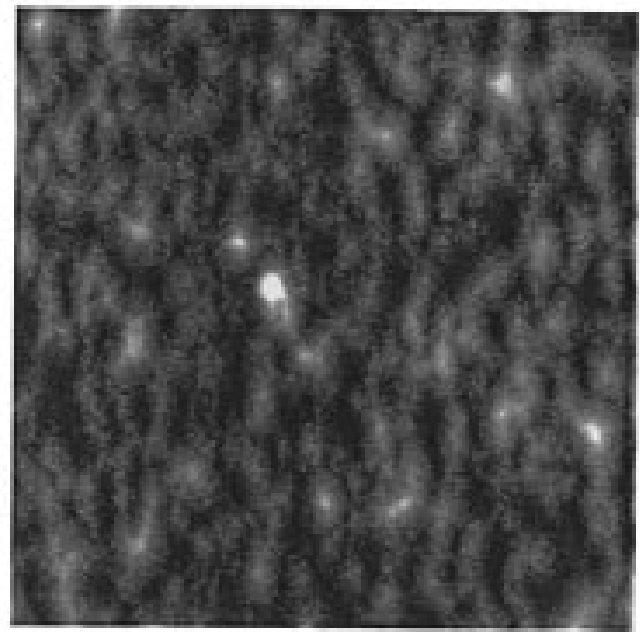

(b)

Fig 3. (a) Continuous phase screen for the Beamlet KPP. The spatial extent is of the image is $50 \mathrm{~cm}$ and the gray scale corresponds to phase values ranging from 0 (black) to 31 radians (white). It was designed using a $256 \times 256$ grid.

Fig. 3 (b) Continuous phase screen for the Nova KPP. The spatial extent is of the image is $70 \mathrm{~cm}$ and the gray scale corresponds to phase values ranging from 0 (black) to 61 radians (white). It was designed using a 512 x 512 grid.
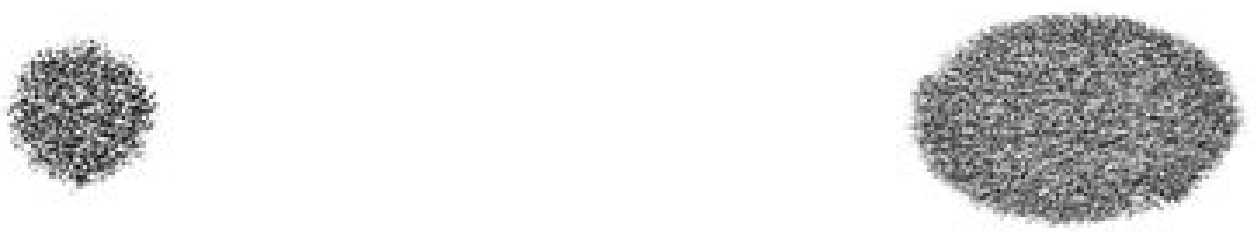

(a)

(b)

Fig 4 (a) Far field intensity produced by the Beamlet KPP shown in fig 3a. For a $7 \mathrm{~m}$ focal length lens and an operating wavelength of $351 \mathrm{~nm}$, the KPP far-field spot diameter is about $350 \mu \mathrm{m}$.

Fig 4 (a) Far field intensity produced by the Nova KPP shown in fig 3b. For a $3 \mathrm{~m}$ focal length lens and an operating wavelength of $351 \mathrm{~nm}$, the major and the minor axes of the ellipse are $400 \mu \mathrm{m}$ and $260 \mu \mathrm{m}$ respectively. 


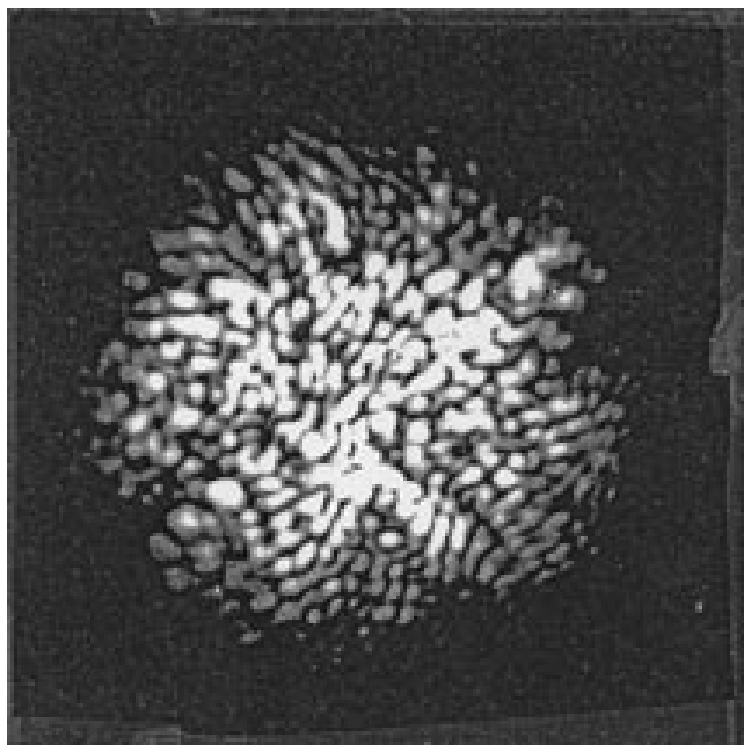

Figure 5. The measured intensity distribution produced by the Beamlet KPP. Approximately $30-\mathrm{cm}$ diameter circular region of the KPP wasilluminated by coherent light at $351 \mathrm{~nm}$. The spot size, scaled to the Beamlet parameters mentioned in fig 4 , is about $350 \mu \mathrm{m}$ in diameter.

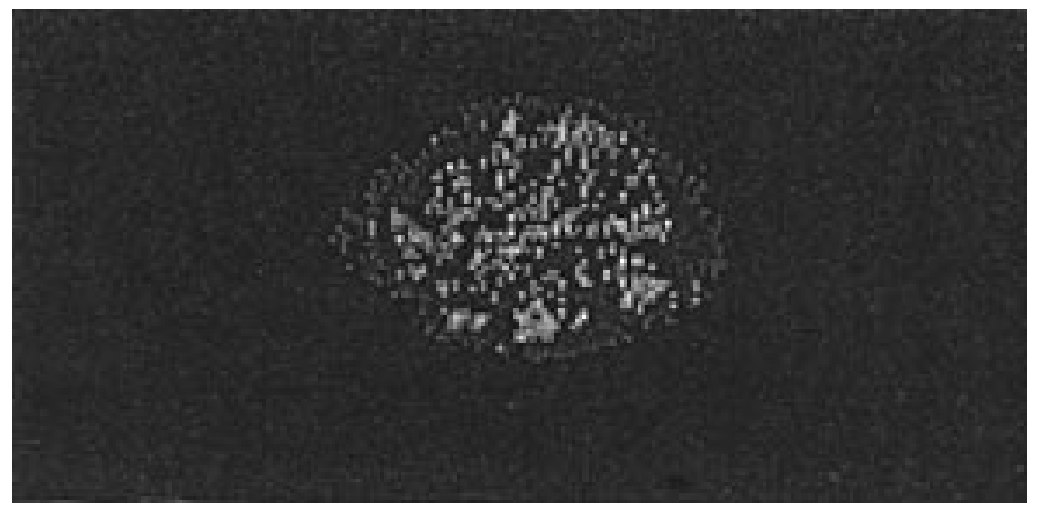

Figure 6. The measured intensity distribution produced by the Nova KPP. Approximately $30-\mathrm{cm}$ diameter circular region of the KPP was illuminated by coherent light at $351 \mathrm{~nm}$. The spot size, scaled to the Nova parameters mentioned in fig 4, is about $400 \times 260 \mu \mathrm{m}$ ellipse. 


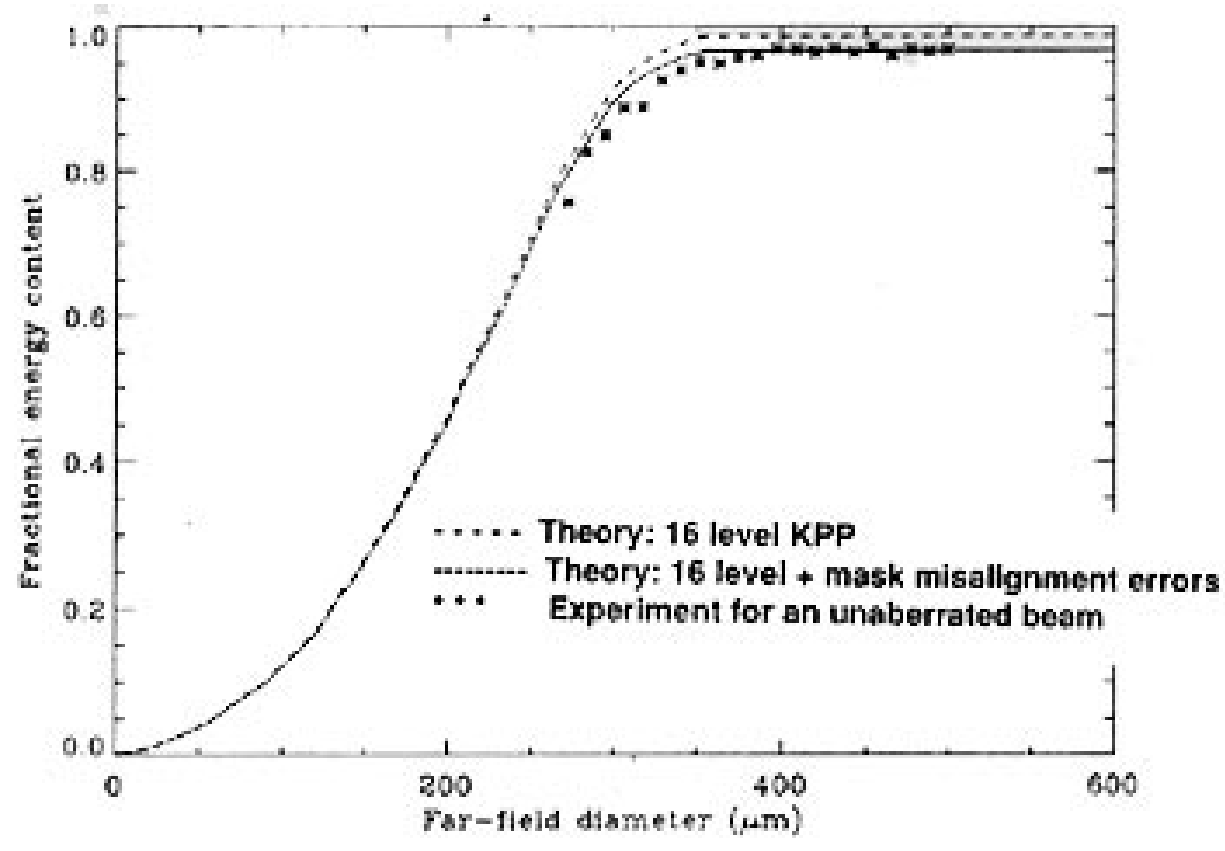

Figure 7. Efficiency measurements of the Beamlet KPP in off-line measurements. The dashed line corresponds to theoretical KPP efficiency, the solid line corresponds to the predicted efficiency taking into account the fabrication errors. The asterrisks indicate maesured efficiencies based on the amount of energy passing through various sized pinholes.

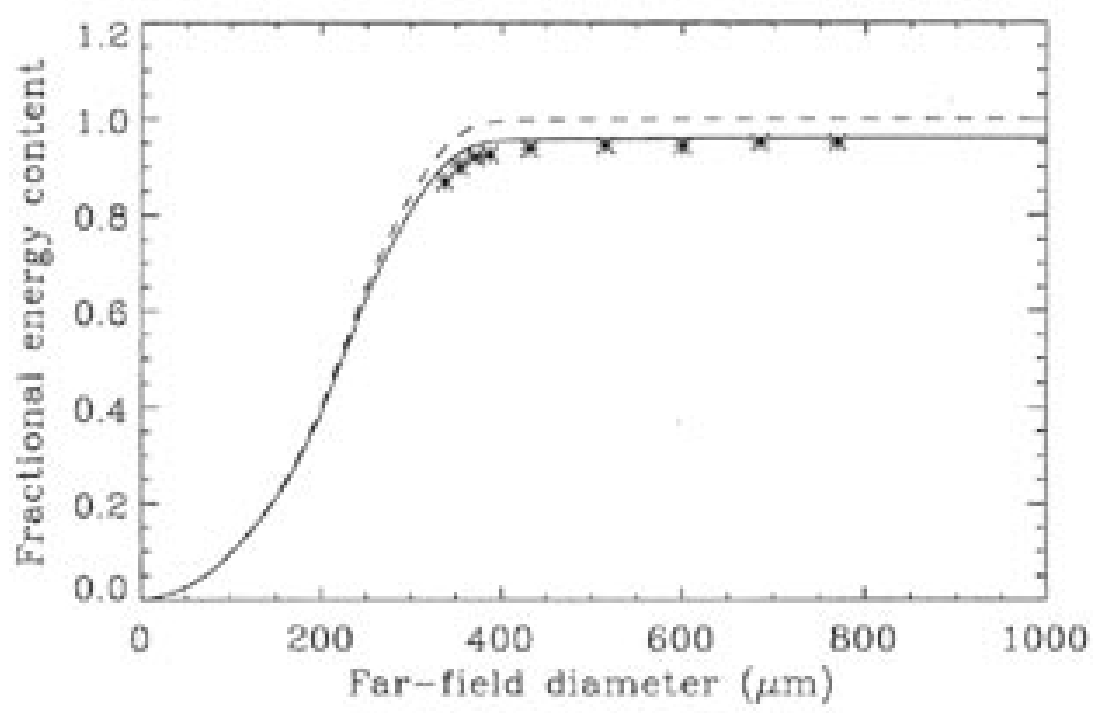

Figure 8. Efficiency measurements of the Nova KPP in off-line measurements. The dashed line corresponds to theoretical KPP efficiency, the solid line corresponds to the predicted efficiency taking into account the fabrication errors. The asterrisks indicate maesured efficiencies based on the amount of energy passing through various sized pinholes. 


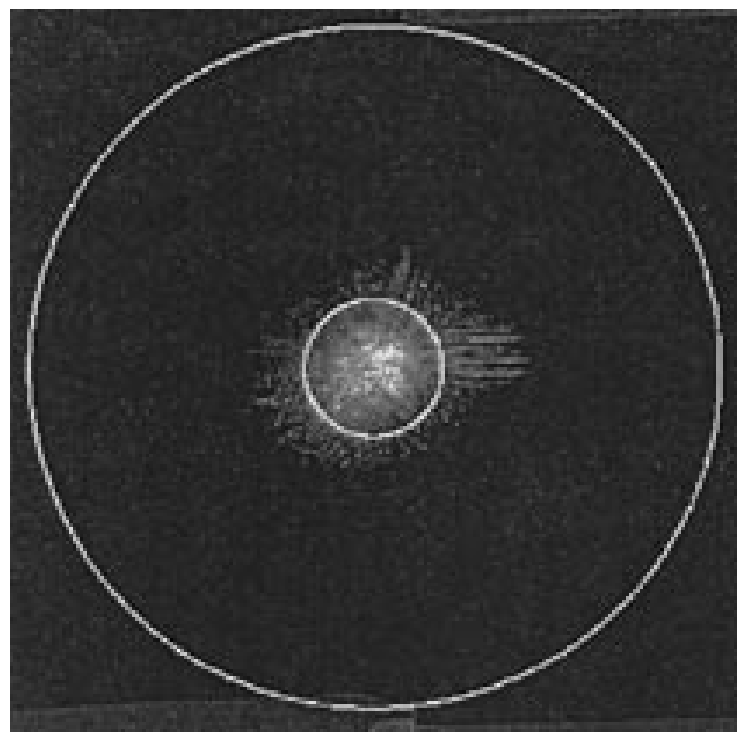

Figure 9. Focal spot produced by the 16 level KPP on the Beamlet laser. The inner circle corresponds to a $600 \mu \mathrm{m}$ diameter spot while the outer circle corresponds to a $3 \mathrm{~m} \mathrm{~m}$ diameter spot.

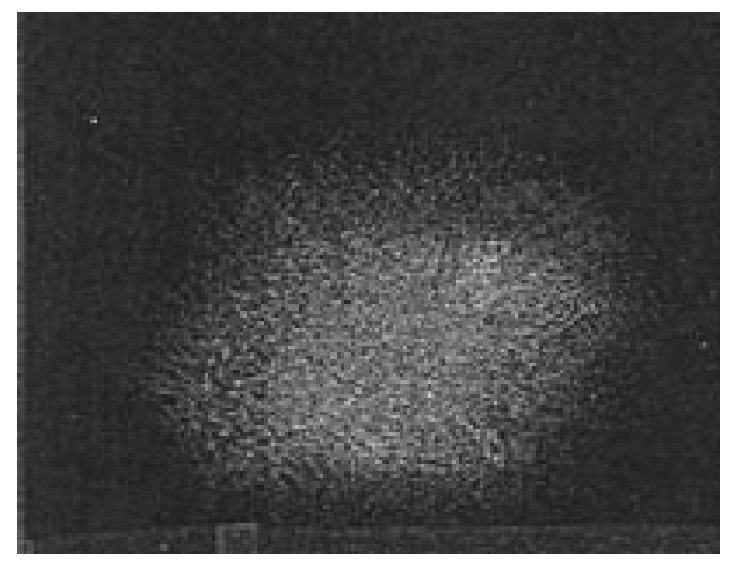

Figure 10. The focal spot produced by the KPP on the Nova laser. The spatial extent of the image is approximately $750 \mu \mathrm{m}$. 


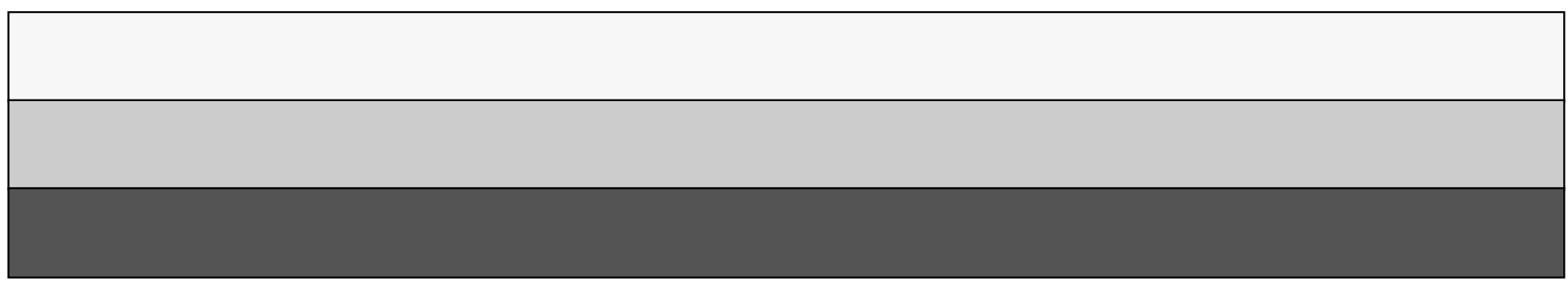

